\title{
Vascular complications in pediatric craniopharyngioma patients: a case-based update
}

\author{
Ulrika Sandvik $^{1}$ (I) $\cdot$ Marcus Ohlsson $^{1} \cdot$ Erik Edström $^{1}$ \\ Received: 19 February 2019 / Accepted: 26 September 2019/Published online: 30 October 2019 \\ (C) The Author(s) 2019
}

\begin{abstract}
Purpose Craniopharyngiomas remain a challenging entity for neurosurgeons because of their deep-seated, midline location, and intimate relationship with critical neurovascular structures. With high long-term survival rates, patients with craniopharyngioma are likely to experience significant late morbidity related to both disease and therapy.

Method and results In this paper, we present two cases of late vascular complications after multi-modal treatment of craniopharyngioma.

Conclusion Available data suggests that pediatric patients with craniopharyngioma represent a particularly vulnerable group.
\end{abstract}

Keywords Craniopharyngioma $\cdot$ Vascular injury $\cdot$ Moyamoya $\cdot$ Fusiform aneurysm $\cdot$ Postradiational vasculopathy

\section{Introduction}

Craniopharyngiomas account for $5-10 \%$ of childhood brain tumors. They are histologically mostly benign, although malignant transformation exists, tumors that arise in the suprasellar region and often affect young patients [1]. Craniopharyngiomas remain a challenging entity for neurosurgeons because of their deep-seated, midline location and intimate relationship with critical neurovascular structures. With high long-term survival rates, patients with craniopharyngioma are likely to experience significant late morbidity related to both disease and therapy. In this paper, we present two of our pediatric cases with childhood-onset craniopharyngioma and development of vascular conditions mandating surgical intervention.

Ulrika Sandvik

ulrika.sandvik@ki.se

Section for Neurosurgery, Department of Clinical Neuroscience, Karolinska Institutet, Stockholm, Sweden

\section{Historical background}

The first reported description of a craniopharyngioma was published in 1857 and the first successful surgical resection of a craniopharyngioma was performed in 1909. For a long period of time, the surgical mortality remained high, according to Harvey Cushing around $14.6 \%$ in 1932. Subsequent reports before 1950 quoted mortality rates between 22 and $41 \%$. The first article reporting vascular complications after craniopharyngioma surgery was a paper reporting cases of carotid artery laceration, vasospasm, and delayed aneurysmal dilatation of the carotid artery [2]. After that, a few case reports have been released but the subject of vascular complications after surgical treatment of craniopharyngioma has not received a lot of attention.

\section{Clinical presentation}

The suprasellar region is surrounded by the carotid arteries, the basilar artery, and branches of both. This proximity to major vessels makes the area particularly vulnerable and there is a high risk of cerebrovascular disease after treatment. Cerebrovascular abnormalities have been attributed to both radiotherapy (RT) and the surgery itself [3]. The clinical 
presentation depends on whether vascular abnormalities result in overt symptoms. Vascular malformations can develop without symptoms and be discovered in routine radiological follow-up as well as present with symptoms of ischemic stroke or hemorrhage [4].

\section{Early vascular complications}

Vascular laceration, arterial occlusion, and arterial vasospasms (sometimes causing infarctions) have been described after transcranial and transsphenoidal surgeries $[2,5,6]$. The importance of subclinical injury to the carotid artery especially in the development of fusiform dilatations of the carotid is well described in the literature [2]. It has been speculated that the development of fusiform dilatations could be caused by a focal arterial wall weakening after dissection of the tumor and that it might be a consequence of injury to the vasa vasorum and that it is more common among centers that aim for total resection [2, 7]. Radiographic abnormalities in the arterial structure have been described to occur as early as 6 to 12 months after craniopharyngioma surgery [2].

However, there is still no certain way to predict which patients will develop late vascular complications.

\section{Late vascular complications}

Radiation has been shown to decrease the recurrence rate of craniopharyngioma to $21 \%$ [8]. This benefit comes at a high price since the radiation itself is known to cause neurocognitive and neuropsychological impairments [8-10]. Radiation has also been described to induce vascular injuries such as degeneration of internal elastic tissue, endothelial damage, and degradation of the vascular wall [1]. The first cases of radiation-induced vasculopathy were reported in 1967 and since then more than 50 cases have been reported $[3,11]$.

Proton beam therapy has been used increasingly to craniopharyngiomas, and radiation-induced large vessel cerebral vasculopathy has been reported in a few patients. A recent study states the incidence of vasculopathy to be approximately $6.7 \%$ and that the median time to development is 1.5 years [12]. Whether there is a difference between the long-term incidence of vasculopathy after proton beam therapy compared with that of photon radiation still remains unclear.

The development of fusiform dilatations on the internal carotid artery after surgical removal of craniopharyngiomas is well described $[2,3,7,13,14]$. One paper describes the fusiform dilatation in as many as $29 \%$ of patients younger than 18 years who have undergone surgery for craniopharyngioma [2] This has later been contradicted by a large multicenter material that states that fusiform dilatations of the carotid occur in $2.4 \%$ of surgically treated childhood-onset adamantinomatous craniopharyngioma [14]. Fusiform dilatations have been described to have a low risk for radiographical progress and the indications for therapy of asymptomatic dilatations are still unclear [2, 14].

Regarding other vascular complications, the development of pseudoaneurysms and aneurysms has been described several years after brain irradiation $[3,15]$. Aneurysm development seems less common compared with vascular stenosis development after radiation therapy [15].

Moyamoya syndrome in children with craniopharyngioma has been described in several cases. Moyamoya disease is an idiopathic disease entity characterized by progressive intracranial occlusion of the anterior circulation followed by the appearance of an abnormal collateral vascular network [1].

Many studies attribute the moyamoya changes to RT whereas certain papers state there might be a genetic relation [4, 16-18]. A median latency of 3 years post-RT has been stated [17]. A study on childhood craniopharyngioma in Boston identified moyamoya in $9 \%$ of the children who had received RT [19]. A recent review reported a total of 76 pediatric cases with postradiational moyamoya [17].

\section{Diagnosis}

Most of the vascular complications seem to be diagnosed on routine follow-up. Long-term effects in children manifesting as stroke, lacunar lesions, stenosis of the middle cerebral artery, internal carotid artery, anterior cerebral artery, cavernomas, and moyamoya syndrome have been described $[1,15,17]$. MR angiograms should also be included in the routine follow-up, and since many of the vascular complications give strokelike symptoms, the threshold for performing perfusion imaging should be low [13].

\section{Management}

The management of vascular complications of craniopharyngioma should be done in a multi-modal setting and the treatment and the follow-up should be individualized.

Fusiform dilatations have been described to have a low risk for radiographical progress and the indications for therapy of asymptomatic dilatations still seem to be unclear $[2,14]$. Due to the expected uneventful clinical course, a conservative approach with radiological follow-ups has been advised [7]. However, a few cases with rapid progression in size have been described. In these nine cases, clipping, endovascular coiling, and bypass surgery have been performed [7, 20-24]. With the advent of endovascular flow diverters, this approach has gained popularity with promising results [25]. 
Postradiation aneurysms seem to have a higher rupture rate; Maruyama et al. state it to be as high as $71 \%$, with the rupture being fatal in $67 \%$ of the cases [26]. In these cases, a more proactive attitude towards treatment is warranted.

In cases of moyamoya syndrome, a direct or indirect surgical revascularization should be considered.

\section{Prognosis and outcomes}

Morbidity after craniopharyngioma surgery is significant, often due to hypothalamic injury, and the risk of recurrence has been described to be as high as $27 \%$, often prompting the need for repeated interventions. Up to $30 \%$ of pediatric craniopharyngioma patients have been described to have vascular abnormalities [13]. In general, patients with craniopharyngioma have a higher cardiovascular mortality in comparison to the general population [14]. In addition, the metabolic syndrome often prevalent among patients with childhood-onset craniopharyngioma also increases the risk of cerebrovascular events [2].

Furthermore, patients with craniopharyngioma treated with RT experience a high rate of stroke compared with the normal population $[15,27]$. The rates of vascular abnormalities and stroke increase with time after RT [15]. The cumulative incidence of moyamoya syndrome after RT for brain tumors has been described to be $14.8 \%$ at 7 years of disease [17]. These numbers are consistent with a Korean study stating a crude incidence of post-RT moyamoya syndrome of $13.3 \%$ [28].

The long-term prognosis of pediatric vascular complications of craniopharyngioma is uncertain. Individualized treatment and follow-up plans seem essential in the management of these patients.

\section{Patient cases}

\section{Patient 1}

Our patient is a young girl born in 1998, who at the age of 5 was diagnosed with a cystic craniopharyngioma causing secondary obstructive hydrocephalus (Fig. 1a). She underwent a transnasal endoscopic fenestration of the cyst and the residual solid component was treated with a single fraction Gamma Knife lesion in 2004. After the RT, she was doing well, attending normal level school, having a normal vision and normal hormonal balance.

Seven years later, a routine follow-up MRI revealed a tumor progression and she was given proton beam irradiation of 54 Gy. Despite the irradiation, a cystic progression was noted the following year and another transnasal endoscopic cyst fenestration was performed.
Two years later, yet another cystic progression was detected. An Ommaya reservoir was placed stereotactically into the cyst via Kocher's point and the right lateral ventricle, to start intracystic interferon-alpha treatment. She developed an unusual adverse effect to this treatment and experienced a sudden visual impairment. An acute MRI showed chiasmatic edema (Fig. 1b). This complication, possibly linked to interferon, has been described by us in a previous publication [29]. Since a pre-treatment leakage test was performed without any signs of leakage outside the cyst, it is possible that the symptoms could be due to a late postradiational effect. Alternatively, an allergic/inflammatory response to the preservative metacresol could be considered. Meta-cresol is a preservative that has been linked to both local and systemic allergic reactions and to local inflammatory reactions in muscle tissue. Eventually, her vision improved slightly, but the visual acuity remained worse compared with that before the start of the intracystic treatment.

At the age of 17, 12 years after the initial diagnosis, she developed a sudden headache. MRI imaging including MRA described a fusiform aneurysm at the superior part of the right carotid siphon (Fig. 1c). A contrast enhancement in the vessel wall was visible and due to this, a vasculitis treatment was initiated. Follow-up MR imaging revealed an unchanged picture despite the steroid treatments. Over the course of 8 months, a slight growth of the aneurysm was noticed, and the patient was treated with an endovascular flow diverter (Fig. 1d-f). Ten months later, a new conventional angiography revealed a persistent contrast circulation in all of the aneurysm. Six months later, she was retreated with a SILK $4 \times$ 30 flow diverter in the carotid siphon and coils in the cranial part of the fusiform aneurysm. The most recent follow-up, digital subtraction angiogram (DSA) 6 months after treatment shows a slight reduction of the aneurysm. She is scheduled for a new follow-up with MR angiogram within 6 months.

\section{Patient 2}

Our second patient is a girl born in 2006. She was diagnosed with a cystic craniopharyngioma at the age of 7 (Fig. 2a). She initially underwent an endoscopic cyst puncture via a frontal burr hole. The procedure had to be repeated 3 weeks later due to insufficient fenestration. The solid tumor components were removed via transsphenoidal endoscopic surgery. Due to postoperative leakage of cerebrospinal fluid, she underwent two revision surgeries. During the immediate postoperative period, the clinical status indicated possible vasospasm. A DSA was performed and presented a normal appearance of the right carotid siphon and middle cerebral artery (Fig. 2b). 
Fig. 1 a Coronal T1-weighted MRI demonstrating initial craniopharyngioma with hydrocephalus. b FLAIR demonstrating chiasmatic edema (arrow and insert). c T1-weighted gadolinium-enhanced MRI demonstrating vessel wall enhancement and aneurysmal dilatation of the right carotid siphon (arrow, insert). $\mathbf{d}-\mathbf{f}$ DSA showing aneurysmal dilatation of the right carotid siphon (d and insert), which was treated in the same setting with a SILK $4 \times$ 30 flow diverter in the carotid siphon and coils in the cranial part of the fusiform aneurysm (e and $\mathbf{f}$, respectively)
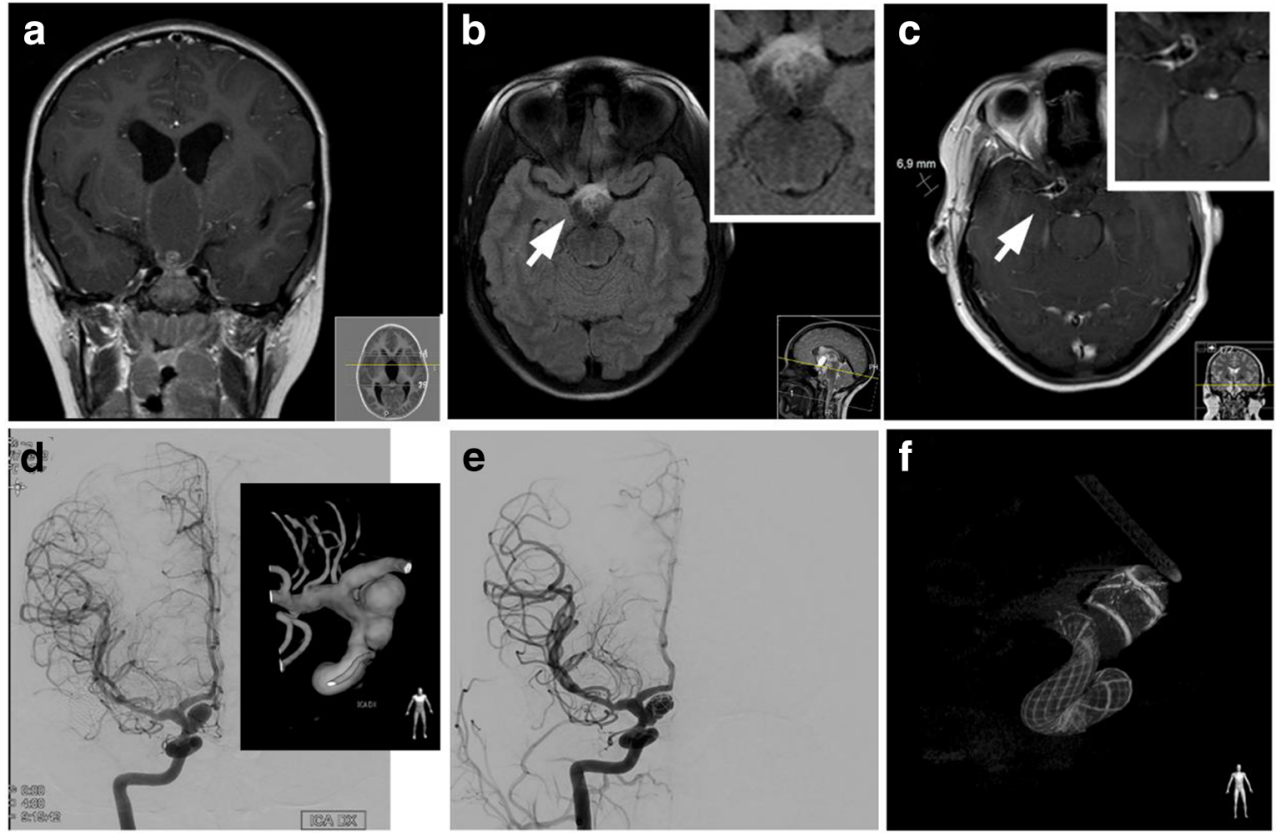

Follow-up MRI revealed a progress in cyst sizes and a stereotactic gamma knife lesion was performed 1 year after the initial diagnosis.

At the age of 10, 3 years after the initial diagnosis, she developed intermittent dysarthria as well as left-sided hemidystonia. MRI including MRA and MR perfusion revealed a right-sided carotid stenosis and a vascular pattern resembling moyamoya syndrome (Fig. 2c). The MR perfusion visualized reduced blood flow in the right hemisphere. A DSA revealed a complete occlusion of the right carotid artery and vascular supply from choroidal collateral vessels (Fig. 2d, e).
Fig. 2 a Coronal T1-weighted gadolinium-enhanced MRI showing initial appearance of the craniopharyngioma. b Frontal DSA demonstrating normal anatomy in the carotid siphon and middle cerebral artery on the right side. $\mathbf{c}$ MRA depicting stenosis of the middle cerebral artery. $\mathbf{d}$ Frontal DSA of the right ICA, please note stenosis of the right carotid siphon and MCA. e Frontal DSA of the right ECA demonstrating dural collaterals supplying the right hemisphere. $\mathbf{f}$ Axial CT showing hemorrhagic infarction in the right thalamus
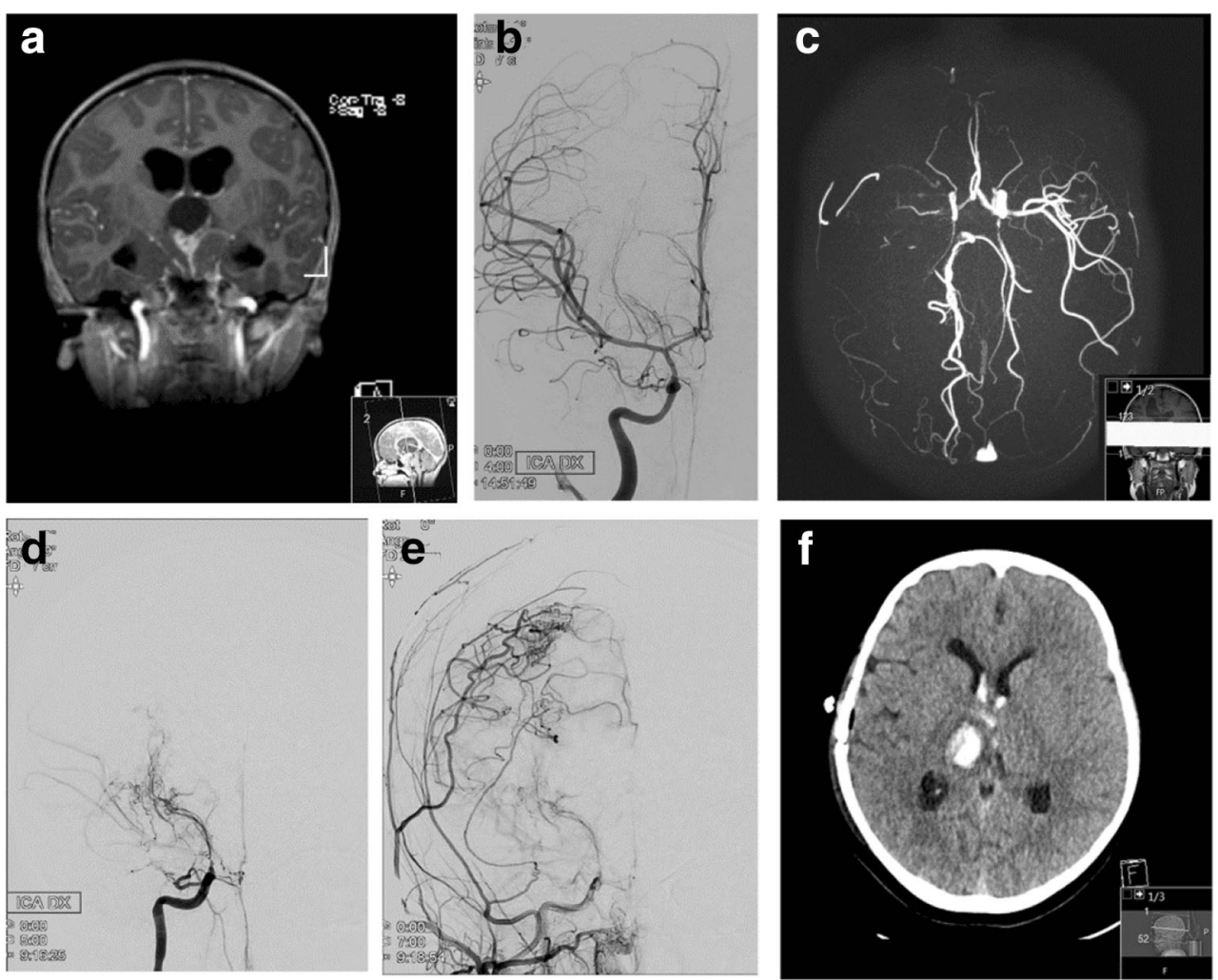
The girl underwent a right-sided temporal artery pial synangiosis in an uneventful procedure. Postoperatively, she displayed a contralateral hemiparesis and computed tomography could verify a hemorrhagic infarction in the left thalamus (Fig. 2f). This infarction is believed to have been caused by blood pressure fluctuations during anesthesia and surgery. Today, 18 months after the surgery, she has recovered from her hemidystonia but still suffers impaired sensibility in her left side.

\section{Summary}

We present two pediatric cases of late vascular complications after multi-modal treatment of craniopharyngioma and review the relevant literature. Available data suggest that pediatric patients with craniopharyngioma represent a particularly vulnerable group. Several factors seem to interact to increase the risk of vascular complications. These include the relative vulnerability of the vessel wall in young patients, the proximity of craniopharyngiomas to the circle of Willis, and repeated exposure of the vessels to potentially harmful treatments including surgery and radiation therapy. More research is needed to elucidate the key steps in the progression of late vascular injury. Nonetheless, the development of late vascular complications in pediatric craniopharyngioma presents a formidable clinical challenge. Clinicians need to reflect on their choices of treatment and be vigilant to the possibility of late vascular complications.

Funding information Open access funding provided by Karolinska Institute.

\section{Compliance with ethical standards}

Conflict of interest On behalf of all authors, the corresponding author states that there is no conflict of interest.

Open Access This article is distributed under the terms of the Creative Commons Attribution 4.0 International License (http:// creativecommons.org/licenses/by/4.0/), which permits unrestricted use, distribution, and reproduction in any medium, provided you give appropriate credit to the original author(s) and the source, provide a link to the Creative Commons license, and indicate if changes were made.

\section{References}

1. Ujifuku K, Matsuo T, Takeshita T, Hayashi Y, Hayashi K, Kitagawa N, Hayashi T, Suyama K, Nagata I (2010) Malignant transformation of craniopharyngioma associated with moyamoya syndrome. Neurol Med Chir (Tokyo) 50:599-603

2. Sutton LN (1994) Vascular complications of surgery for craniopharyngioma and hypothalamic glioma. Pediatr Neurosurg 21(Suppl 1):124-128
3. Pereira P, Cerejo A, Cruz J, Vaz R (2002) Intracranial aneurysm and vasculopathy after surgery and radiation therapy for craniopharyngioma: case report. Neurosurgery 50:885-887 discussion $887-888$

4. Smith ER (2015) Structural causes of ischemic and hemorrhagic stroke in children: moyamoya and arteriovenous malformations. Curr Opin Pediatr 27:706-711

5. Hoffman HJ, De Silva M, Humphreys RP, Drake JM, Smith ML, Blaser SI (1992) Aggressive surgical management of craniopharyngiomas in children. J Neurosurg 76:47-52

6. Yasargil MG, Curcic M, Kis M, Siegenthaler G, Teddy PJ, Roth P (1990) Total removal of craniopharyngiomas. Approaches and long-term results in 144 patients. J Neurosurg 73:3-11

7. Wang L, Shi X, Liu F, Qian H (2016) Bypass surgery to treat symptomatic fusiform dilation of the internal carotid artery following craniopharyngioma resection: report of 2 cases. Neurosurg Focus 41:E17

8. Thompson D, Phipps K, Hayward R (2005) Craniopharyngioma in childhood: our evidence-based approach to management. Child's Nerv Syst : ChNS : official journal of the International Society for Pediatric Neurosurgery 21:660-668

9. Kalapurakal JA (2005) Radiation therapy in the management of pediatric craniopharyngiomas-a review. Child's Nerv Syst : ChNS : official journal of the International Society for Pediatric Neurosurgery 21:808-816

10. Bailey S, Parkes J (2015) Intracystic interferon therapy in childhood craniopharyngioma: who, when and how? Clin Endocrinol 82:29-34

11. Darmody WR, Thomas LM, Gurdjian ES (1967) Postirradiation vascular insufficiency syndrome Case report. Neurology 17:1190 1192

12. Kralik SF, Watson GA, Shih C-S, Ho CY, Finke W, Buchsbaum J (2017) Radiation-induced large vessel cerebral vasculopathy in pediatric patients with brain tumors treated with proton radiation therapy. Int J Radiat Oncol*Biol*Physics 99(4):817-824

13. Liu AK, Bagrosky B, Fenton LZ, Gaspar LE, Handler MH, McNatt SA, Foreman NK (2009) Vascular abnormalities in pediatric craniopharyngioma patients treated with radiation therapy. Pediatr Blood Cancer 52:227-230

14. Hoffmann A, Warmuth-Metz M, Lohle K, Reichel J, Daubenbuchel AM, Sterkenburg AS, Muller HL (2016) Fusiform dilatation of the internal carotid artery in childhood-onset craniopharyngioma: multicenter study on incidence and long-term outcome. Pituitary 19: 422-428

15. Lo AC, Howard AF, Nichol A, Hasan H, Martin M, Heran M, Goddard K (2016) A cross-sectional cohort study of cerebrovascular disease and late effects after radiation therapy for craniopharyngioma. Pediatr Blood Cancer 63:786-793

16. Ganesan V, Smith ER (2015) Moyamoya: defining current knowledge gaps. Dev Med Child Neurol 57:786-787

17. Wu YH, Chang FC, Liang ML, Chen HH, Wong TT, Yen SH, Chen YW (2016) Incidence and long-term outcome of postradiotherapy moyamoya syndrome in pediatric patients with primary brain tumors: a single institute experience in Taiwan. Cancer Med 5:21552160

18. Parray T, Martin TW, Siddiqui S (2011) Moyamoya disease: a review of the disease and anesthetic management. J Neurosurg Anesthesiol 23:100-109

19. Winkfield KM, Tsai HK, Yao X, Larson E, Neuberg D, Pomeroy SL, Ullrich NJ, Cohen LE, Kieran MW, Scott RM, Goumnerova LC, Marcus KJ (2011) Long-term clinical outcomes following treatment of childhood craniopharyngioma. Pediatr Blood Cancer $56: 1120-1126$

20. Lakhanpal SK, Glasier CM, James CA, Angtuaco EJ (1995) MR and CT diagnosis of carotid pseudoaneurysm in children following surgical resection of craniopharyngioma. Pediatr Radiol 25:249-251 
21. Nagata T, Goto T, Ichinose T, Mitsuhashi Y, Tsuyuguchi N, Ohata K (2010) Pathological findings of fusiform dilation of the internal carotid artery following radical dissection of a craniopharyngioma. J Neurosurg Pediatr 6:567-571

22. Ogilvy CS, Tawk RG, Mokin M, Yang X, Levy EI, Hopkins LN, Siddiqui AH (2011) Stent-assisted coiling treatment of pediatric traumatic pseudoaneurysm resulting from tumor surgery. Pediatr Neurosurg 47:442-448

23. Tirakotai W, Sure U, Benes L, Aboul-Enein H, Schulte DM, Riegel T, Bertalanffy H (2002) Successful management of a symptomatic fusiform dilatation of the internal carotid artery following surgery of childhood craniopharyngioma. Child's Nerv Syst : ChNS : official journal of the International Society for Pediatric Neurosurgery 18: 717-721

24. Li Q, Wang C, Xu J, You C (2015) Endovascular treatment for fusiform dilation of internal carotid artery following craniopharyngioma resection: a case illustration. J Child Neurol 30:1354-1356

25. Rajah G, Narayanan S, Rangel-Castilla L (2017) Update on flow diverters for the endovascular management of cerebral aneurysms. Neurosurg Focus 42:E2
26. Maruyama K, Mishima K, Saito N, Fujimaki T, Sasaki T, Kirino T (2000) Radiation-induced aneurysm and moyamoya vessels presenting with subarachnoid haemorrhage. Acta Neurochir 142:139-143

27. Campen CJ, Kranick SM, Kasner SE, Kessler SK, Zimmerman RA, Lustig R, Phillips PC, Storm PB, Smith SE, Ichord R, Fisher MJ (2012) Cranial irradiation increases risk of stroke in pediatric brain tumor survivors. Stroke 43:3035-3040

28. Lee HS, Seol HJ, Kong DS, Shin HJ (2011) Moyamoya syndrome precipitated by cranial irradiation for craniopharyngioma in children. J Korean Neurosurg Soc 50:535-537

29. Sandvik U, Grillner P, Holm S, Gustavsson B (2016) Interferon or late effect of radiotherapy? Child's Nerv Syst : ChNS : official journal of the International Society for Pediatric Neurosurgery 32: 229-230

Publisher's note Springer Nature remains neutral with regard to jurisdictional claims in published maps and institutional affiliations. 